\title{
Modelling of freezing kinetics of extract of betel leaves (Piper betle L.)
}

\begin{abstract}
Betel is a native medicinal plant from central and eastern parts of Peninsular Malaysia. It is now an important commercial crop in India and Sri Lanka. There are various beneficial bioactivities discovered in this herb including anti-carcinogenic, anti-inflammatory, antioxidant, and antimicrobial properties. Freeze drying is one of the common methods used to dehydrate the herbal aqueous extract. This work studied the freezing process of aqueous extract of betel leaves (Piper betle L.). The effects of different freezing temperatures on the freezing kinetics were investigated. Freezing temperatures of $-20,-25,-30^{\circ} \mathrm{C}$ were selected for the study. The freezing time which was taken as the time required for the temperature of the sample to reach the freezing temperature was determined from the kinetics data. The freezing point of betel leaves extract was determined as $-4^{\circ} \mathrm{C}$. The freezing process was described with numerical model. The predicted data showed good agreement with the experimental results.
\end{abstract}

Keyword: Betel leaf; Liquid freezing; Freezing kinetics; Freezing time; Numerical model 